\title{
COMPOSIÇÃO E ESTRUTURA DE COMUNIDADES ARBÓREAS DE CERRADO stricto sensu NO NORTE DO TOCANTINS E SUL DO MARANHÃO ${ }^{1}$
}

\author{
Marcelo Brilhante de Medeiros² e Bruno Machado Teles Walter ${ }^{2}$
}

\begin{abstract}
RESUMO - Este trabalho teve por objetivo avaliar a composição e a estrutura da comunidade arbórea de cerrado stricto sensu (s.s.) no município de Filadélfia (norte do Tocantins) e compará-la com a mesma fitofisionomia em uma área adjacente na bacia do rio Tocantins, no município de Carolina (MA), na província norte-nordeste do Cerrado. Em Filadélfia foram alocadas aleatoriamente 10 parcelas de $20 \times 50 \mathrm{~m}$, totalizando um ha. Todos os indivíduos lenhosos com diâmetro de caule $\geq 5 \mathrm{~cm}$, medidos a $30 \mathrm{~cm}$ do solo, foram incluídos. Os dados de composição e estrutura da vegetação em Carolina foram obtidos em literatura. Dois métodos de análise foram empregados: UPGMA, adotando-se o índice de Sørensen, e TWINSPAN, agrupando parcelas e espécies. Em Filadélfia foram encontrados 789 indivíduos, 53 espécies e 44 gêneros; H' foi igual a 3,32 nats. ind ${ }^{-1} \mathrm{e}$ J'=0,83. Nove espécies (Qualea parviflora, Pouteria ramiflora, Curatella americana, Hirtella ciliata, Qualea grandiflora, Parkia platycephala, Diospyros sericea, Stryphnodendron sp. e Stryphnodendron rotundifolium) representaram 49,9\% do VI total. Das 69 espécies encontradas nas duas áreas, 36 foram comuns. O índice de Sørensen mostrou baixa similaridade florística entre Carolina e Filadélfia. A análise por TWINSPAN também mostrou similaridade qualitativa e quantitativa reduzidas entre as áreas. Os resultados revelaram que as comunidades de cerrado s.s. são diferenciadas entre e dentre as áreas, com provável influência das diferenças de solo. Não obstante, a fitossociologia foi similar a de outros estudos na região e a composição é característica da província fitogeográfica norte/nordeste do bioma Cerrado, contendo elementos típicos como Hirtella ciliata, Platonia insignis e Caryocar coriaceum.
\end{abstract}

Palavras-chave: Fitossociologia, Diversidade de espécies e Similaridade.

\section{COMPOSITION AND STRUCTURE OF THE WOODY CERRADO stricto sensu IN NORTHERN TOCANTINS AND SOUTHERN MARANHÃO}

\begin{abstract}
The objective of this work was to evaluate the composition and structure of the woody community of cerrado stricto sensu fragments located in Carolina (southern Maranhão), and Filadelfia (northern Tocantins) in the north-northeastern phytogeographic Cerrado province. The survey was carried out with ten $1000 \mathrm{~m}^{2}$ plots $(20 \times 50 \mathrm{~m})$ at Filadélfia. The composition and structure data of the same vegetation physiognomy from Carolina was obtained by using literature. The Sørensen Index with UPGMA linkage method and the TWINSPAN were used to evaluate the similarities between the woody communities. All wood individuals showing diameters $\geq 5 \mathrm{~cm}$, at $30 \mathrm{~cm}$ above the ground level were recorded. In Filadelfia county 789 individuals, 53 species and 44 genus were recorded. Nine species (Qualea parviflora, Pouteria ramiflora, Curatella americana, Hirtella ciliata, Qualea grandiflora, Parkia platycephala, Diospyros sericea, Stryphnodendron sp. and Stryphnodendron rotundifolium) reached $49.9 \%$ of the total VI; $H^{\prime}=3.32$ and $J^{\prime}=0.83$. Among the total of 69 species, 36 species were found in the both counties. The Sфrensen Index has shown low floristic similarity between Carolina and Filadélfia. Also the TWINSPAN has shown low qualitative and quantitative similarity between and within areas. These results have shown differences between and within areas of cerrado stricto sensu that most likely
\end{abstract}

\footnotetext{
${ }^{1}$ Recebido em 09.10.2011 e aceito para publicação em 28.05.2012.

${ }^{2}$ Embrapa Recursos Genéticos e Biotecnologia, Núcleo Temático de Recursos Genéticos-Herbário.E-mail:<marcelo.brilhante@embrapa.br> e $<$ bruno.walter@embrapa.br>.
} 


\begin{abstract}
was associated with the different types of soils. The phytosociological results were similar to other surveys around the region and the floristic composition is typical of the north/northeastern phytogeographic Cerrado province. Typical species from this Cerrado province were recorded such as Hirtella ciliata, Platonia insignis and Caryocar coriaceum.
\end{abstract}

Keywords: Phytosociology, Species diversity and Similarity.

\section{INTRODUÇÃO}

O conceito amplo de Cerrado engloba trechos de vegetação onde faltam os elementos arbóreos (campos) até aqueles onde há o domínio de árvores (o Cerradão). Entre esses extremos fisionômicos, no sentido mais difundido de Coutinho (1978), com o aumento dos elementos lenhosos, estão incluídos o Campo Sujo, o Campo Cerrado e o Cerrado stricto sensu (EITEN, 1994a; RIBEIRO; WALTER, 2008).

A flora do bioma Cerrado é composta basicamente pelas mesmas famílias e gêneros que ocorrem na Mata Atlântica e na Amazônia, mas as espécies são distintas e com distribuição heterogênea dentro do bioma, além das proporções diferentes dos gêneros e espécies (RATTER et al., 2003; BRIDGEWATER et al., 2004). Adicione-se a isso, conforme Forzza et al. (2010), que $29,7 \%$ das plantas com sementes endêmicas do Brasil, ou 4.151 espécies, pertencem ao bioma Cerrado. Com base em 951 espécies lenhosas amostradas em 376 levantamentos ao longo do bioma, Ratter et al. (2003) indicaram sete províncias fitogeográficas para o Cerrado lato sensu. Das espécies analisadas, mais da metade é encontrada em apenas uma província, com poucas espécies comuns a todas elas (RATTER et al., 2003; BRIDGEWATER et al., 2004). Dos 376 levantamentos analisados por esses autores, a maioria se concentrou diretamente em trechos de Cerrado stricto sensu.

Conforme Ribeiro e Walter (2008), o Cerrado stricto sensu se caracteriza pela presença de árvores baixas, inclinadas, tortuosas, com ramificações irregulares e geralmente com evidências de queimadas. Apresenta o estrato herbáceo-subarbustivo espalhado e exuberante no período chuvoso. Já os troncos das plantas lenhosas possuem ritidoma composto por cortiça espessa, suas folhas são geralmente rígidas e coriáceas, caracterizando xeromorfismo. Os referidos autores sugeriram quatro subdivisões dentro dessa fitofisionomia, que diferem em gradiente de densidade arbórea desde o Cerrado
Denso, passando pelo Cerrado Típico, o Cerrado Ralo até o Cerrado Rupestre, sendo este último de ocorrência em áreas de afloramentos rochosos e, portanto, caracterizado pelo tipo de substrato.

A região Nordeste do Estado do Tocantins e o Sudoeste do Maranhão ainda apresentam remanescentes bem preservados de Cerrado stricto sensu, embora essas áreas estejam sendo rapidamente convertidas para fins agropecuários (WALTER; AQUINO, 2004; AQUINO et al., 2007a; MEDEIROS; WALTER, 2008). Particularmente, a bacia do rio Tocantins apresenta elevada diversidade de espécies lenhosas de Cerrado (RATTER et al., 2003). Apesar da representatividade, o conhecimento sobre a composição e estrutura das comunidades arbóreas do Cerrado stricto sensu naquela região é muito incipiente (MEDEIROS; WALTER, 2008). As informações se restringem a um relevante estudo florístico geral levado a termo por Eiten (1994b), levantamentos pontuais como o de Walter e Aquino (2004) e estudos em poucos locais no Maranhão, como Balsas (AQUINO et al., 2007ab), Santa Quitéria (IMAÑAENCINAS et al., 1995; PAULA et al., 1998) e Carolina (MEDEIROS; WALTER, 2008).

Os objetivos deste trabalho foram avaliar e comparar a composição e estrutura da comunidade arbórea de remanescentes de Cerrado stricto sensu no Município de Filadélfia (Norte do Tocantins) e compará-las com dados publicados sobre a mesma fitofisionomia em área adjacente, no Município de Carolina, MA.

\section{MATERIAL E MÉTODOS}

\subsection{Caracterização das áreas}

O estudo foi conduzido no Município Filadélfia (TO), entre as coordenadas $07^{\circ} 17^{\prime} 35^{\prime \prime}$ S e $47^{\circ} 35^{\prime}$ '29", $\mathrm{W}$, em altitudes de 150 a $170 \mathrm{~m}$. A área pertence à bacia do médio rio Tocantins e situa-se em uma região ecotonal entre dois dos maiores biomas sul-americanos: a Floresta Amazônica e o Cerrado. 
A bacia do rio Tocantins, naquela região, incluindo as duas áreas, enquadra-se em um regime de chuvas nitidamente tropical, em que o período chuvoso responde por mais de $80 \%$ do total anual precipitado (CESTE, 2004). A temperatura média anual registrada pela Estação Climatológica de Carolina é de $26,1^{\circ} \mathrm{C}$, distante cerca de $50 \mathrm{~km}$ de Filadélfia, com precipitação média de $1.718,7 \mathrm{~mm}$ (CESTE, 2004).

As classes de solos em cada área foram obtidas com base no mapa de solos para os Estados do Tocantins (SEPLAN, 2008) e do Maranhão (EMBRAPA, 2011). A classe de solo que predomina nas duas áreas amostradas é o Neossolo Quartzarênico, em topografia plana (SEPLAN, 2008; EMBRAPA, 2011). Porém, ocorrem diferenças dentro e entre os solos das duas áreas, e em Filadélfia ocorrem também Neossolos Litólicos (SEPLAN, 2008), enquanto em Carolina ocorrem, além de solos litólicos, os Gleissolos, Plintossolos, Podzólicos e Latossolos (EMBRAPA, 2011).

A área amostral é coberta por Cerrado stricto sensu (RIBEIRO; WALTER, 2008), com predomínio do subtipo Típico ocorrendo em mosaicos com Cerrado ralo.

\subsection{Amostragem}

Nos fragmentos de Cerrado stricto sensu em Filadélfia foram alocadas aleatoriamente 10 parcelas de $20 \times 50 \mathrm{~m}$ (1,0 ha), com distância mínima de $50 \mathrm{~m}$, totalizando 1,0 ha amostrado. Os dados de composição e estrutura de Cerrado stricto sensu do Município de Carolina foram obtidos por Medeiros e Walter (2008).

Embora o solo em cada parcela não tenha sido caracterizado do ponto de vista físico-químico, considerou-se que os dois municípios possuíam composição de solos distinta, com base nas classes de solo (SEPLAN, 2008; EMBRAPA, 2011), as quais influenciam a composição e estrutura da vegetação.

O levantamento fitossociológico incluiu todos os indivíduos lenhosos, exceto lianas, com diâmetro a $30 \mathrm{~cm}$ do solo $\left(\right.$ DAS $\left._{30 \mathrm{~cm}}\right) \geq 5 \mathrm{~cm}$. Os diâmetros foram mensurados com a utilização de suta graduada em $\mathrm{cm}$. Em troncos irregulares, elípticos, duas medidas foram tomadas em campo (nos ângulos retos de cada uma), e a média é que foi registrada. No caso de troncos ramificados abaixo de $30 \mathrm{~cm}$ do solo, duas ou mais medidas foram tomadas separadamente e o diâmetro quadrático foi calculado. As alturas totais foram estimadas visualmente, sempre pelo mesmo observador.
Os parâmetros fitossociológicos densidade, frequência e dominância (relativas e absolutas), além do valor de importância (VI), foram calculados por meio do programa Mata Nativa (CIENTEC, 2004). Para estimar a riqueza de espécies foram utilizados dois métodos não paramétricos (COLWELL; CONDDINGTON, 1994): 1) jackknife, $1^{\mathrm{a}}$ e $2^{\mathrm{a}}$ ordens; e 2) bootstrap. Os estimadores de riqueza foram calculados com o auxílio do programa Estimates v.7.5 (COLWELL, 2005). A diversidade florística para a amostragem fitossociológica foi analisada pelo índice de Shannon H' (base do logaritmo neperiano/indivíduo) e a equitabilidade, pelo índice de Pielou (J') (KREBS, 1989).

Para realizar as comparações entre as comunidades nas áreas, foram feitas análises multivariadas com métodos de agrupamento. $\mathrm{Na}$ análise de similaridade florística, o Índice de Sørensen foi calculado pelo programa MVSP 3.13 (KOVACH, 2007) e, para a formação dos agrupamentos, foi utilizado o método de ligação Unweighted Pair Group Method with Arithmetic Mean UPGMA. A composição e estrutura da vegetação nas parcelas foram comparadas e classificadas por TWINSPAN Two Way Indicator Species Analysis (HILL, 1979), utilizando o programa PC-ORD, versão 3.17 (McCUNE; MEFFORD, 1997). Os agrupamentos gerados por essa classificação são considerados estatisticamente fortes se seus autovalores forem superiores a 0,3 (HILL, 1979).

$\mathrm{Na}$ florística, as espécies foram agrupadas em famílias, segundo o APG II (2003), e o material testemunho está depositado no Herbário CEN, da Embrapa Recursos Genéticos e Biotecnologia, em Brasília, Distrito Federal (DF).

\section{RESULTADOS}

Nas duas áreas foram registrados 69 espécies, 57 gêneros e 28 famílias, das quais 36 espécies foram comuns a ambas (Tabela 1). Isso significa que quase a metade das espécies (33) foi encontrada em apenas uma das áreas amostradas. O índice de Shannon calculado para Carolina foi de 3,04 nats/indivíduo, com equitabilidade (J') de 0,77 (MEDEIROS; WALTER, 2008), enquanto para Filadélfia H' foi um pouco superior, com 3,32 nats/indivíduo e J' igual a 0,83.

No Município de Carolina foram encontrados 542 ind.ha $^{-1}$, pertencentes a 52 espécies, 45 gêneros e 25 famílias. Sete espécies (Byrsonima crassa, Curatella 
Tabela 1 - Táxons amostrados em 2 ha de Cerrado stricto sensu nos Municípios de Carolina, MA; e Filadelfia, TO. Famílias sensu APG II (2003).

Table 1 - Surveyed taxa in two hectares of Cerrado stricto sensu in Carolina (MA) and Filadelfia (TO) counties. Botanic families by APG II (2003).

\begin{tabular}{|c|c|c|c|}
\hline Família & Gêneros/espécies & MA & $\mathrm{TO}$ \\
\hline \multirow[t]{2}{*}{ Anacardiaceae } & Anacardium occidentale L. & - & $\mathrm{x}$ \\
\hline & Astronium fraxinifolium Schott ex Spreng. & $\mathrm{x}$ & $\mathrm{x}$ \\
\hline Annonaceae & Xylopia aromatica (Lam.) Mart. & $\mathrm{x}$ & $\mathrm{x}$ \\
\hline \multirow[t]{2}{*}{ Bignoniaceae } & Handroanthus ochraceus (Cham.) Mattos & $\mathrm{x}$ & $\mathrm{x}$ \\
\hline & Handroanthus serratifolius (A.H.Gentry) S.Grose & $\mathrm{x}$ & - \\
\hline Caryocaraceae & Caryocar coriaceum Witmm. & $\mathrm{x}$ & $\mathrm{x}$ \\
\hline \multirow[t]{2}{*}{ Chrysobalanaceae } & Couepia cf. grandiflora (Mart. \& Zucc.) Benth. ex Hook.f. & $\mathrm{x}$ & $\mathrm{x}$ \\
\hline & Hirtella ciliata Mart. \& Zucc. & $\mathrm{x}$ & $\mathrm{x}$ \\
\hline \multirow[t]{2}{*}{ Clusiaceae } & Kielmeyera coriacea Mart. \& Zucc. & - & $\mathrm{x}$ \\
\hline & Platonia insignis Mart. & $\mathrm{x}$ & - \\
\hline \multirow[t]{2}{*}{ Combretaceae } & Combretum duarteanum Cambess. & $\mathrm{x}$ & - \\
\hline & Terminalia sp. & $\mathrm{x}$ & - \\
\hline \multirow[t]{2}{*}{ Connaraceae } & Connarus suberosus Planch. & $\mathrm{x}$ & $\mathrm{x}$ \\
\hline & Rourea induta Planch. & $\mathrm{x}$ & $\mathrm{x}$ \\
\hline Dilleniaceae & Curatella americana $\mathrm{L}$. & $\mathrm{x}$ & $\mathrm{x}$ \\
\hline \multirow[t]{2}{*}{ Ebenaceae } & Diospyros sericea A. DC. & $\mathrm{x}$ & $\mathrm{x}$ \\
\hline & Diospyros hispida DC. & - & $\mathrm{x}$ \\
\hline Erythroxylaceae & Erythroxylum suberosum St. Hil. & - & $\mathrm{x}$ \\
\hline \multirow[t]{19}{*}{ Fabaceae (Leguminosae) } & Senegalia sp. & $\mathrm{x}$ & - \\
\hline & Andira nitida Mart. ex Benth. & $\mathrm{x}$ & $\mathrm{x}$ \\
\hline & Andira vermifuga Mart. ex Benth. & - & $\mathrm{x}$ \\
\hline & Bauhinia longifolia (Bong.) Steudel & $\mathrm{x}$ & - \\
\hline & Dimorphandra gardneriana Tul. & $\mathrm{x}$ & $\mathrm{x}$ \\
\hline & Dimorphandra mollis Benth. & $\mathrm{x}$ & $\mathrm{x}$ \\
\hline & Hymenaea stigonocarpa Mart. ex Hayne & - & $\mathrm{x}$ \\
\hline & Leptolobium dasycarpum Vogel & $\mathrm{x}$ & $\mathrm{x}$ \\
\hline & Machaerium aculeatum Raddi & $\mathrm{x}$ & - \\
\hline & Machaerium acutifolium Vogel & - & $\mathrm{x}$ \\
\hline & Parkia platycephala Benth. & - & $\mathrm{x}$ \\
\hline & Plathymenia reticulata Benth. & $\mathrm{x}$ & $\mathrm{x}$ \\
\hline & Pterodon emarginatus Vogel & $\mathrm{x}$ & - \\
\hline & Tachigali aurea Tul. & $\mathrm{x}$ & $\mathrm{x}$ \\
\hline & Tachigali vulgaris L.G.Silva \& H.C.Lima & $\mathrm{x}$ & $\mathrm{x}$ \\
\hline & Stryphnodendron rotundifolium Mart. & $\mathrm{x}$ & $\mathrm{x}$ \\
\hline & Stryphnodendron sp. & - & $\mathrm{x}$ \\
\hline & Swartzia sp. & - & $\mathrm{x}$ \\
\hline & Vatairea macrocarpa (Benth.) Ducke & $\mathrm{x}$ & $\mathrm{x}$ \\
\hline Icacinaceae & Emmotum nitens (Benth.) Miers & $\mathrm{x}$ & - \\
\hline Lythraceae & Lafoensia pacari A. St.-Hil. & $\mathrm{x}$ & $\mathrm{x}$ \\
\hline \multirow[t]{4}{*}{ Malpighiaceae } & Byrsonima coccolobifolia Kunth & $\mathrm{x}$ & $\mathrm{x}$ \\
\hline & Byrsonima crassa Nied. & $\mathrm{x}$ & $\mathrm{x}$ \\
\hline & Byrsonima sp. & - & $\mathrm{x}$ \\
\hline & Heteropterys byrsonimiifolia A. Juss. & $\mathrm{x}$ & $\mathrm{x}$ \\
\hline \multirow[t]{2}{*}{ Malvaceae } & Eriotheca gracilipes (K. Schum.) A. Robyns & - & $\mathrm{x}$ \\
\hline & Luehea divaricata Mart. & $\mathrm{x}$ & $\mathrm{x}$ \\
\hline \multirow[t]{2}{*}{ Melastomataceae } & Mouriri elliptica Mart. & $\mathrm{x}$ & - \\
\hline & Mouriri pusa Gardner & $\mathrm{x}$ & $\mathrm{x}$ \\
\hline Moraceae & Brosimum gaudichaudii Trécul & - & $\mathrm{x}$ \\
\hline
\end{tabular}

Revista Árvore, Viçosa-MG, v.36, n.4, p.673-683, 2012 
Tabela 1 - Cont.

\begin{tabular}{|c|c|c|c|}
\hline Família & Gêneros/espécies & MA & $\mathrm{TO}$ \\
\hline \multirow[t]{3}{*}{ Myrtaceae } & Eugenia cf. aurata $\mathrm{O}$. Berg & $\mathrm{x}$ & - \\
\hline & Myrcia rostrata DC. & $\mathrm{x}$ & - \\
\hline & Psidium guyanense Pers. & $\mathrm{x}$ & $\mathrm{x}$ \\
\hline Ochnaceae & Ouratea hexasperma (A. St.-Hil.) Baill. & $\mathrm{x}$ & $\mathrm{x}$ \\
\hline Opiliaceae & Agonandra brasilienses Miers ex Benth. \& J.D. Hook. & $\mathrm{x}$ & - \\
\hline \multirow[t]{5}{*}{ Rubiaceae } & Cordiera concolor (Cham.) Kuntze & - & $\mathrm{x}$ \\
\hline & Coussarea hydrangeifolia (Benth.) Benth. \& Hook.f. ex Müll. Arg. & - & $\mathrm{x}$ \\
\hline & Ferdinandusa elliptica Pohl & - & $\mathrm{x}$ \\
\hline & Guettarda pohliana Müll. Arg. & $\mathrm{x}$ & - \\
\hline & Tocoyena formosa (Cham \& Schltdl.) K. Schum. & $\mathrm{x}$ & $\mathrm{x}$ \\
\hline Salicaceae & Casearia sylvestris $\mathrm{Sw}$. & - & $\mathrm{x}$ \\
\hline Sapindaceae & Magonia pubescens A. St.-Hil. & $\mathrm{x}$ & $\mathrm{x}$ \\
\hline \multirow[t]{2}{*}{ Sapotaceae } & Pouteria ramiflora (Mart.) Radlk. & $\mathrm{x}$ & $\mathrm{x}$ \\
\hline & Pouteria torta (Mart.) Radlk & $\mathrm{x}$ & $\mathrm{x}$ \\
\hline Simaroubaceae & Simarouba versicolor A. St.-Hil. & $\mathrm{x}$ & $\mathrm{x}$ \\
\hline Urticaceae & Cecropia pachystachya Trécul. & $\mathrm{x}$ & - \\
\hline \multirow[t]{5}{*}{ Vochysiaceae } & Callisthene fasciculata Mart. & $\mathrm{x}$ & $\mathrm{x}$ \\
\hline & Qualea grandiflora Mart. & $\mathrm{x}$ & $\mathrm{x}$ \\
\hline & Qualea parviflora Mart. & $\mathrm{x}$ & $\mathrm{x}$ \\
\hline & Salvertia convallariaeodora A. St.-Hil. & $\mathrm{x}$ & - \\
\hline & Vochysia rufa Mart. & $\mathrm{x}$ & $\mathrm{x}$ \\
\hline
\end{tabular}

americana, Callisthene fasciculata, Qualea parviflora, Salvertia convallariaeodora, Hirtella ciliata e Qualea grandiflora) representaram cerca de $50 \%$ do VI total (MEDEIROS; WALTER, 2008). Os valores de riqueza estimados pelo método jackknife foram de 71,9 e 78,7 espécies, para $1^{\mathrm{a}}$ e $2^{\mathrm{a}}$ ordens, respectivamente. No método bootstrap foram estimadas 62 espécies (MEDEIROS; WALTER, 2008).

No Município de Filadélfia foram encontrados 789 ind. ha $^{-1}$, pertencentes a 53 espécies, 44 gêneros e 24 famílias (Tabelas 1 e 2). Nove espécies (Qualea parviflora, Pouteria ramiflora, Curatella americana, Hirtella ciliata, Qualea grandiflora, Parkia platycephala, Diospyros sericea, Stryphnodendron sp. e Stryphnodendron rotundifolium) representaram cerca de $50 \%$ do VI total (Tabela 2). Os valores de riqueza estimados pelo método jackknife foram de 68,4 e 72,6 espécies, para $1^{\mathrm{a}}$ e $2^{\mathrm{a}}$ ordens, respectivamente. No método bootstrap, foi estimado um total de 61 espécies.

$\mathrm{Na}$ amostra de Carolina, nove indivíduos foram encontrados mortos em pé, representando $1,7 \%$ do total amostrado (MEDEIROS; WALTER, 2008). Em Filadélfia, 20 foram encontrados mortos em pé, totalizando em $2,5 \%$ da densidade relativa.
A curva espécies-área para a amostra de Carolina apresentou tendência à estabilização, uma vez que, na metade da amostra, 73,3\% das espécies já haviam sido registradas (MEDEIROS; WALTER, 2008). O mesmo ocorreu em Filadélfia, com 80,2\% das espécies incluídas em apenas 0,5 ha.

A análise pelo método de ligação UPGMA separou os grupos de parcelas em cada município, com valores menores que $50 \%$ de similaridade, evidenciando as diferenças florísticas entre as áreas (Figura 1). Em Filadélfia, as parcelas de 1 a 6 apresentaram agrupamento com valores superiores a $50 \%$ de similaridade, enquanto as parcelas 7, 8 e 10 formaram um segundo grupo distinto, com valores próximos a 50\%. Em Carolina, as parcelas $11,12,13$ e 14 mostraram os maiores valores de similaridade, acima de $50 \%$. As parcelas 17 e 18 formaram um grupo distinto, com similaridade menor do que $10 \%$ em relação às demais, caracterizando um Cerrado onde se destacaram as espécies Callisthene fasciculata, Curatella americana e Senegalia sp.

Na análise pelo método TWINSPAN, com autovalor significativo na primeira divisão, foram separadas as parcelas 17 e 18 das demais (Figura 2). A partir da terceira divisão, com autovalor significativo, as parcelas de

Revista Árvore, Viçosa-MG, v.36, n.4, p.673-683, 2012 
Tabela 2 - Espécies amostradas em 1 ha de Cerrado stricto sensu no Município de Filadélfia, TO. Valores em ordem decrescentes pelo Valor de Importância (VI), em que D = Densidade (n/ha), Do = Dominância $\left(\mathrm{m}^{2} / \mathrm{ha}\right), \mathrm{F}=$ Frequência $(\%)$, $\mathrm{A}=$ absoluta, $\mathrm{R}=$ relativa e Hmax = altura máxima de indivíduos da espécie $(\mathrm{m})$.

Table 2 - Species sampled from one hectare of cerrado stricto sensu in Filadelfia county, TO. Decreasing values by Importance Value $(V I) . D=$ Density $(n / h a), D o=$ Dominance $(\mathrm{m} 2 / \mathrm{ha}), F=$ Frequency $(\%), A=$ Absolute, $R=$ Relative and Hmax = Maximum individual height per species.

\begin{tabular}{|c|c|c|c|c|c|c|c|c|}
\hline Nome Científico & $\mathrm{DA}$ & DR & FA & FR & DoA & DoR & VI & Hmáx. \\
\hline Qualea parviflora & 100 & 12,67 & 100 & 5,24 & 0,657 & 6,9 & 24,81 & 8,3 \\
\hline Pouteria ramiflora & 56 & 7,1 & 70 & 3,66 & 1,05 & 11,02 & 21,782 & 11 \\
\hline Curatella americana & 45 & 5,7 & 90 & 4,71 & 0,823 & 8,65 & 19,061 & 7,9 \\
\hline Hirtella ciliata & 38 & 4,82 & 100 & 5,24 & 0,499 & 5,24 & 15,29 & 8,8 \\
\hline Qualea grandiflora & 48 & 6,08 & 80 & 4,19 & 0,462 & 4,85 & 15,124 & 6,2 \\
\hline Parkia platycephala & 32 & 4,06 & 60 & 3,14 & 0,699 & 7,34 & 14,541 & 9,4 \\
\hline Diospyros sericea & 49 & 6,21 & 70 & 3,66 & 0,341 & 3,58 & 13,457 & 5,2 \\
\hline Stryphnodendron sp. & 40 & 5,07 & 80 & 4,19 & 0,374 & 3,93 & 13,184 & 8,7 \\
\hline Stryphnodendron rotundifolium & 38 & 4,82 & 80 & 4,19 & 0,323 & 3,39 & 12,398 & 9,5 \\
\hline Caryocar coriaceum & 20 & 2,53 & 50 & 2,62 & 0,61 & 6,4 & 11,552 & 12 \\
\hline Anacardium occidentale & 17 & 2,15 & 60 & 3,14 & 0,524 & 5,51 & 10,802 & 9 \\
\hline Plathymenia reticulata & 29 & 3,68 & 60 & 3,14 & 0,305 & 3,2 & 10,019 & 9,5 \\
\hline Andira vermifuga & 13 & 1,65 & 50 & 2,62 & 0,528 & 5,54 & 9,806 & 10,4 \\
\hline Callisthene fasciculata & 35 & 4,44 & 30 & 1,57 & 0,283 & 2,97 & 8,981 & 7,5 \\
\hline Plantas mortas & 20 & 2,53 & 60 & 3,14 & 0,299 & 3,13 & 8,81 & 5 \\
\hline Lafoensia pacari & 31 & 3,93 & 30 & 1,57 & 0,129 & 1,36 & 6,855 & 4,2 \\
\hline Tocoyena formosa & 16 & 2,03 & 80 & 4,19 & 0,055 & 0,58 & 6,798 & 4,5 \\
\hline Ouratea hexasperma & 14 & 1,77 & 60 & 3,14 & 0,12 & 1,26 & 6,174 & 5,2 \\
\hline Byrsonima crassa & 23 & 2,92 & 30 & 1,57 & 0,096 & 1 & 5,488 & 4 \\
\hline Tachigali aurea & 9 & 1,14 & 60 & 3,14 & 0,048 & 0,5 & 4,784 & 8,9 \\
\hline Byrsonima coccolobifolia & 8 & 1,01 & 50 & 2,62 & 0,092 & 0,97 & 4,598 & 7,7 \\
\hline Diospyros hispida & 8 & 1,01 & 30 & 1,57 & 0,121 & 1,27 & 3,859 & 5,8 \\
\hline Tachigali vulgaris & 5 & 0,63 & 10 & 0,52 & 0,21 & 2,2 & 3,358 & 9,5 \\
\hline Erythroxylum suberosum & 5 & 0,63 & 40 & 2,09 & 0,027 & 0,29 & 3,015 & 3,1 \\
\hline Magonia pubescens & 7 & 0,89 & 20 & 1,05 & 0,082 & 0,86 & 2,797 & 9,7 \\
\hline Luehea divaricata & 8 & 1,01 & 20 & 1,05 & 0,07 & 0,74 & 2,798 & 5,5 \\
\hline Mouriripusa & 5 & 0,63 & 30 & 1,57 & 0,053 & 0,56 & 2,761 & 7,25 \\
\hline Couepia cf. grandiflora & 8 & 1,01 & 20 & 1,05 & 0,057 & 0,6 & 2,661 & 6 \\
\hline Astronium fraxinifolium & 3 & 0,38 & 30 & 1,57 & 0,053 & 0,56 & 2,507 & 7,5 \\
\hline Vochysia rufa & 6 & 0,76 & 20 & 1,05 & 0,064 & 0,67 & 2,476 & 7,5 \\
\hline Dimorphandra gardneriana & 4 & 0,51 & 30 & 1,57 & 0,014 & 0,14 & 2,22 & 4 \\
\hline Rourea induta & 6 & 0,76 & 20 & 1,05 & 0,025 & 0,26 & 2,068 & 3,6 \\
\hline Connarus suberosus & 5 & 0,63 & 20 & 1,05 & 0,025 & 0,27 & 1,946 & 3,7 \\
\hline Xylopia aromatica & 4 & 0,51 & 20 & 1,05 & 0,033 & 0,35 & 1,901 & 6,5 \\
\hline Psidium guyanensis & 3 & 0,38 & 20 & 1,05 & 0,043 & 0,45 & 1,875 & 4,7 \\
\hline Simarouba versicolor & 3 & 0,38 & 20 & 1,05 & 0,027 & 0,29 & 1,715 & 5,5 \\
\hline Handroanthus ochraceus & 3 & 0,38 & 20 & 1,05 & 0,017 & 0,18 & 1,605 & 4,4 \\
\hline Dimorphandra mollis & 2 & 0,25 & 20 & 1,05 & 0,022 & 0,24 & 1,536 & 4,6 \\
\hline Andira nitida & 2 & 0,25 & 20 & 1,05 & 0,012 & 0,12 & 1,422 & 3,8 \\
\hline Byrsonima sp. & 5 & 0,63 & 10 & 0,52 & 0,017 & 0,18 & 1,332 & 3,4 \\
\hline Leptolobium dasycarpum & 1 & 0,13 & 10 & 0,52 & 0,055 & 0,57 & 1,225 & 12,5 \\
\hline Brosimum gaudichaudii & 1 & 0,13 & 10 & 0,52 & 0,05 & 0,53 & 1,178 & 6,2 \\
\hline Vatairea macrocarpa & 2 & 0,25 & 10 & 0,52 & 0,036 & 0,37 & 1,151 & 5,5 \\
\hline Eriotheca gracilipes & 1 & 0,13 & 10 & 0,52 & 0,026 & 0,27 & 0,923 & 6,1 \\
\hline Cordiera concolor & 2 & 0,25 & 10 & 0,52 & 0,01 & 0,1 & 0,88 & 4,05 \\
\hline Hymenaea stignocarpa & 1 & 0,13 & 10 & 0,52 & 0,018 & 0,19 & 0,836 & 5,2 \\
\hline Pouteria torta & 1 & 0,13 & 10 & 0,52 & 0,011 & 0,12 & 0,767 & 6 \\
\hline Kielmeyera coriacea & 1 & 0,13 & 10 & 0,52 & 0,012 & 0,13 & 0,781 & 4,7 \\
\hline Swartzia sp. & 1 & 0,13 & 10 & 0,52 & 0,003 & 0,03 & 0,685 & 1,5 \\
\hline Coussarea hydrangeifolia & 1 & 0,13 & 10 & 0,52 & 0,003 & 0,03 & 0,679 & 2,7 \\
\hline Machaerium acutifolium & 1 & 0,13 & 10 & 0,52 & 0,004 & 0,04 & 0,69 & 3,5 \\
\hline Casearia sylvestris & 1 & 0,13 & 10 & 0,52 & 0,003 & 0,03 & 0,679 & 2,75 \\
\hline Heteropterys byrsonimiifolia & 1 & 0,13 & 10 & 0,52 & 0,003 & 0,03 & 0,682 & 3,05 \\
\hline Ferdinandusa elliptica & 1 & 0,13 & 10 & 0,52 & 0,003 & 0,03 & 0,678 & 3,9 \\
\hline Totais & 789 & 100 & 1910 & 100 & 9,525 & 100 & 300 & 12,5 \\
\hline
\end{tabular}

Revista Árvore, Viçosa-MG, v.36, n.4, p.673-683, 2012 


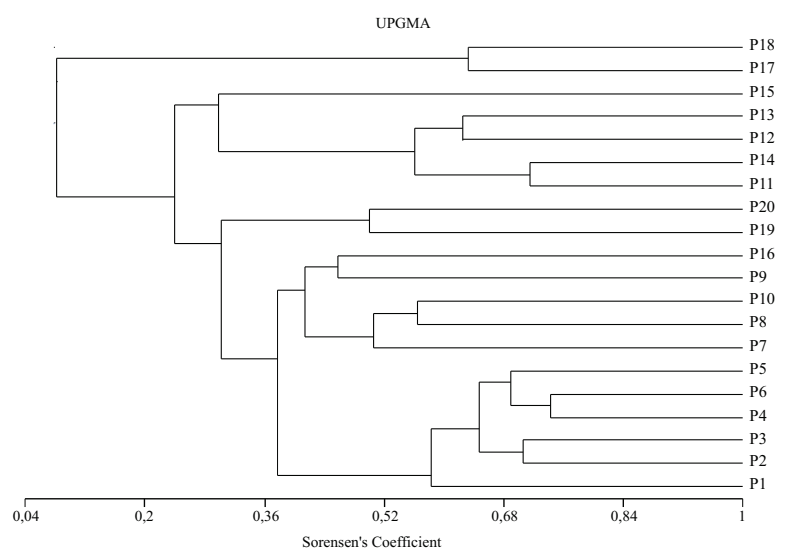

Figura 1 - Dendrograma plotado com base no índice de Sørensen, utilizando análise de agrupamento UPGMA em 20 parcelas alocadas em 2 ha de Cerrado sensu stricto nos município de Filadélfia/TO (P1 - P10) e Carolina/ MA (P11 - P20).

Figure 1 - Dendrogram of S $\phi$ rensen índex by using UPGMA linkage method of 20 samples in two hectares of cerrado sensu stricto in Filadélfia/TO (P1 - P10) and Carolina/MA (P11-P20).

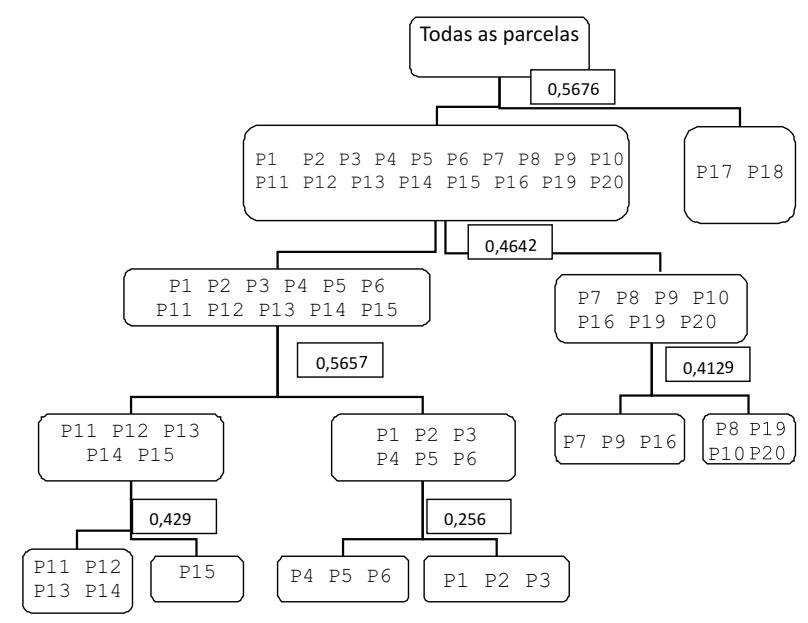

Figura 2 - Análise pelo método TWINSPAN de 20 parcelas alocadas em dois hectares de cerrado stricto sensu nos municípios de Filadélfia/TO (P1 - 10) e Carolina/ MA (P11 - 20). Os retângulos contém os autovalores (eigenvalues) de cada divisão.

Figure 2 - Dendrogram of TWINSPAN method of 20 samples at two hectares of cerrado sensu stricto in Filadélfial TO (P1 - P10) and Carolina/MA (P11 - P20). Rectangles include eingenvalues. cada município formaram grupos mais distintos. A separação foi mais pronunciada no grupo de parcelas 1 a 6 (Filadélfia) e 11 a 15 (Carolina), também reforçando o resultado da similaridade. Nesses agrupamentos, espécies preferenciais de Carolina foram Andira nitida, Emmotum nitens, Leptolobium dasycarpum, Platonia insignis, Pouteria ramiflora e Salvertia convallariaeodora, enquanto no Município de Filadélfia foram preferenciais as espécies Anacardium occidentale, Andira vermifuga, Caryocar coriaceum, Diospyros sericea, Ouratea hexasperma, Parkia platycephala e Tocoyena formosa. A separação das parcelas 7 a 10 das demais parcelas ( 1 a 6) em Filadélfia, assim como a separação das parcelas 16,19 e 20 das demais parcelas em Carolina, também revelou diferenças florísticas e estruturais dentro de cada município.

\section{DISCUSSÃO}

Os valores de riqueza estimados em ambas as áreas foram similares, com maiores valores pelo método jacknife. Esses valores de riqueza se encontram na faixa de variação registrada no Estado do Tocantins, embora não tenham sido verificados valores muito elevados, de até 97 espécies, característicos de algumas áreas mais ricas da bacia do rio Tocantins (RATTER et al., 2003). Em relação aos estimadores, Krebs (1989) e Hellmann e Fowler (1999) sugeriram que, em amostras inferiores a 20 parcelas, as variantes do método jackknife apresentam desempenho superior às estimativas geradas pelo método bootstrap, e esse método resulta em dados mais robustos para amostras maiores. Por essa premissa, é mais provável que a riqueza em espécies local seja mais próxima das estimativas de jackknife.

Com relação às plantas mais importantes amostradas nas duas áreas, regionalmente Aquino et al. (2007a) também registraram elevada densidade de Byrsonima crassa, Pouteria ramiflora, Qualea grandiflora, Qualea parviflora, Salvertia convallariaeodora e Vochysia rufa, que parecem dominar trechos de Cerrado do Sul do Maranhão e fazem parte das espécies frequentes na província norte/nordeste do Cerrado (RATTER et al., 2003; BRIDGEWATER et al., 2004). Hirtella ciliata, outra espécie importante nas duas áreas amostradas, foi indicada por Castro et al. (1998) entre as plantas de ampla distribuição dessa província, que ainda inclui como espécies características Parkia platycephala, Platonia insignis, Dimorphandra gardneriana e Caryocar coriaceum (RATTER et al., 2003; 
BRIDGEWATER et al., 2004; MEDEIROS; WALTER, 2008), também presentes nas áreas amostradas.

Quanto à densidade, os valores de Carolina revelaram-se baixos. Isso sugere um Cerrado aberto, com baixa densidade arbórea, de modo que a caracterização fitofisionômica precisa para o trecho em Carolina seria o subtipo ralo (MEDEIROS; WALTER, 2008). Em Filadélfia, por sua vez, o trecho amostrado encaixa-se no subtipo típico de Ribeiro e Walter (2008). Os números de indivíduos por hectare registrados em publicações são muito variáveis ao longo do bioma, o que expressa as variações naturais encontradas no Cerrado stricto sensu. No Distrito Federal, por exemplo, há desde o registro de apenas 552 ind.ha ${ }^{-1}$ (ROSSI et al., 1998) até 1.396 ind.ha $^{-1}$ (FELFILI et al., 1994; SILVAJÚNIOR; FELFILI, 1998). Valores muito elevados (4.463 ind.ha ${ }^{-1}$ ) foram verificados em Minas Gerais, no Município de Abaeté (SAPORETTI-JUNIOR et al., 2003). Com métodos similares aos deste estudo, Balduino et al. (2005) registraram densidade de 1.990 ind.ha $^{-1}$, no centro do Estado (Paraopeba, MG), o que também é um valor elevado. Já no alto Tocantins, em Goiás, Felfili et al. (2007) registraram 1.271 ind.ha $^{-1} \mathrm{em}$ Serra Negra, e Aquino et al. (2007a) amostraram 1.406 ind.ha ${ }^{-1}$ em Balsas, no Sul do Maranhão. Neste último exemplo, porém, o critério de inclusão de espécies foi DAS $\mathrm{DA}_{30} \geq 3 \mathrm{~cm}$, o que deve ter ampliado a densidade encontrada.

As porcentagens de indivíduos mortos em pé foram baixas, inferiores aos dados registrados em outros estudos, cujos valores variaram entre $3,69 \%$ e $13,59 \%$ (p. ex. NOGUEIRA et al., 2001; FELFILI et al., 2007; FONSECA; SILVA-JÚNIOR, 2004). A ocorrência de fogo e outros distúrbios antrópicos, como o uso de áreas nativas como pastagem para gado, tem sido apontada como causas para os elevados valores de mortalidade e raleamento da camada lenhosa no Cerrado (MEDINA; SILVA, 1990; EITEN, 1994a; AQUINO et al., 2007a). Em ambas as áreas de estudo são comuns tanto a pecuária extensiva quanto o uso de fogo como ferramenta de manejo das pastagens (MEDEIROS; WALTER, 2008). Porém, o impacto dessas atividades antrópicas sobre o Cerrado stricto sensu na região apenas poderá ser avaliado apropriadamente em estudos de longo prazo, os quais incluem dados sobre as taxas de mortalidade e não apenas os dados de indivíduos mortos em pé, podendo os últimos variar muito temporalmente (AQUINO et al., 2007a).
Os valores de diversidade em Filadélfia e em Carolina (MEDEIROS; WALTER, 2008) estão praticamente inclusos no largo intervalo entre 3,07 e 3,78 registrado em diferentes áreas do Cerrado lato sensu, assim como os valores de equitabilidade, entre 0,75 e 0,88 (FELFILI et al., 1997, 2001, 2007; MARIMON et al., 1998; NOGUEIRA et al., 2001; APPOLINARIO; SCHIAVINI, 2002; SAPORETTIJUNIOR et al., 2003; FONSECA; SILVA-JÚNIOR, 2004; BALDUINO etal., 2005; MEDEIROS et al., 2005; AQUINO et al., 2007a).

As análises por TWINSPAN confirmaram as diferenças florísticas evidenciadas pelo índice de Sørensen, corroborando a análise de similaridade qualitativa e separando as parcelas nas duas áreas. Como exemplo de diferenciação florística entre as duas áreas, determinadas espécies só foram encontradas em uma delas. Esse foi o caso de Brosimum gaudichaudii, Casearia sylvestris, Diospyros hispida, Eriotheca gracilipes, Erythroxylum suberosum e Hymenaea stigonocarpa, em Filadélfia (TO); e Kielmeyera coriacea e Salvertia convallariaeodora, em Carolina (MA).

Ainda, esse método revelou maiores diferenças, em comparação com o índice de Sørensen, entre as parcelas de Filadélfia, com separações entre o grupo de parcelas de 1 a 6 do grupo de parcelas 7 a 10 e, também, com a separação das parcelas 16, 19 e 20 em Carolina, como provável resultado da elevada diversidade de solos observada dentro desses municípios (SEPLAN, 2008; EMBRAPA, 2011).

As parcelas 17 e 18, separadas na primeira divisão, caracterizaram-se pela ocorrência de vários indivíduos de Senegalia sp., planta muito associada a ambientes florestais, além de numerosos indivíduos de Callisthene fasciculata, espécie característica de solos mesotróficos (RATTER et al., 2003); e Curatella americana, espécie frequente em todo o bioma Cerrado (BRIGDEWATER et al., 2004).

Os resultados apresentados revelaram que tanto a composição quanto a estrutura das comunidades lenhosas do Cerrado stricto sensu são diferenciadas entre e dentro das duas áreas, refletindo muito provavelmente as diferenças nas classes de solos. Mesmo com a predominância de Neossolos Quartzarênicos e solos litólicos, tanto em Filadélfia quanto em Carolina (MEDEIROS; WALTER, 2008), há outras classes de solos que não são comuns a ambas as áreas, como 
as áreas com Plintossolos, Podzólicos, Gleissolos e Latossolos observadas neste último município, associadas aos Neossolos Quartzarênicos predominantes (SEPLAN, 2008; EMBRAPA, 2011). Assim, as diferenças florísticas e estruturais poderiam ser explicadas pelo "sistema de terras" onde foram alocadas as parcelas de cada município, pois as características ambientais, como as diferentes classes de solos e topografia, representam maior influência na composição e estrutura da vegetação do que a proximidade geográfica (FELFILI et al., 1994).

\section{CONCLUSÃO}

Os remanescentes de Cerrado sensu stricto na região Sul do Maranhão e Norte do Tocantins revelaram padrões de diversidade e riqueza um pouco abaixo de padrões para a bacia do rio Tocantins, mas dentro das faixas de variação para outros estudos, considerando-se o bioma como um todo.

As análises comparativas entre as duas áreas próximas revelaram que as comunidades de Cerrado s.s. são diferenciadas entre as áreas. O "sistema de terras" pode explicar essas diferenças, considerando-se as diferentes classes de solo em cada área. A despeito das diferenças observadas, a composição geral da vegetação em ambas as áreas é característica da província norte/nordeste do bioma e inclui seus elementos típicos, como Hirtella ciliata, Parkia platycephala, Curatella americana, Platonia insignis, Dimorphandra gardneriana e Caryocar coriaceum.

\section{AGRADECIMENTOS}

À Fundação de Apoio à Pesquisa e ao Agronegócio FAGRO e a João Benedito Pereira, Gledson Alves Moreira e Juliene Roveratti, pelo auxílio nas atividades de campo.

\section{REFERÊNCIAS}

APG II. An update of the Angiosperm Phylogeny Group classification for the orders and families of flowering plants: APG II. Botanical Journal of the Linnean Society, v.141, n.4, p.399-436, 2003.

APPOLINARIO, V.; SCHIAVINI, I. Levantamento fitossociológico de espécies arbóreas de cerrado (stricto sensu) em Uberlândia - Minas Gerais. Boletim do Herbário Ezechias Paulo Heringer, v. 10, p.57-75, 2002.
AQUINO, F. G.; WALTER, B. M. T.; RIBEIRO, J. F. Woody community dynamics in two fragments of "cerrado" stricto sensu over a seven-year period (1995-2002), MA, Brazil. Revista Brasileira de Botânica, v.30, n.1, p.113121, 2007a.

AQUINO, F. G.; WALTER, B. M. T.; RIBEIRO, J. F. Dinâmica de populações de espécies lenhosas de Cerrado, Balsas, Maranhão. Revista Árvore, v.31, n.5, p.793-803, 2007b.

BALDUINO, A. P. C. et al. Fitossociologia e análise comparativa da composição florística do Cerrado da flora de Paraopeba - MG. Revista Árvore, v.24, n.1, p.25-34, 2005.

BRIDGEWATER, S.; RATTER, J. A.; RIBEIRO, J. F. Biogeographic patterns, âdiversity and dominance in the cerrado biome of Brazil. Biodiversity and Conservation, v.13, p.2295-2318. 2004.

CASTRO, A. A. J. F.; MARTINS, F. R.; FERNANDES, A. G. The woody flora of the cerrado vegetation in the state of Piauí, northeastern Brazil. Edinburgh Journal of Botany, v.55, p.455-472, 1998.

CESTE. Estudo de Impacto AmbientalRelatório de Impacto Ambiental do AHE Estreito. Rio de Janeiro: Ceste Consórcio Estreito de Energia, 2004. 235p.

CIENTEC. Mata Nativa - Sistema para análise fitossociológica e elaboração de planos de manejo de florestas nativas. Viçosa, MG: 2004.

COLWELL, R. K. EstimateS: Statistical estimates of species richness and shared species from samples. Version 7.5. Persistent URL $<$ purl.oclc.org/estimates>, 2005.

COLWELL, R. K.; CODDINGTON, J. A. Estimating terrestrial biodiversity through extrapolation. Philosophical Transactions of the Royal Society (Series B), v.345, p.101-118, 1994.

Revista Árvore, Viçosa-MG, v.36, n.4, p.673-683, 2012 
COUTINHO, L. M. O conceito de Cerrado. Revista Brasileira de Botânica, v.1, n.1, p.17-23, 1978.

EITEN, G. Vegetação do Cerrado In: PINTO, M. N. (Ed.). Cerrado: caracterização, ocupação e perspectivas. 2.ed. Brasília: UnB/SEMATEC, 1994a. p.17-73.

EITEN, G. Duas travessias na vegetação do Maranhão. Brasília: UnB, 1994b. 76p.

EMPRESA BRASILEIRA DE PESQUISA AGROPECUÁRIA - EMBRAPA. Sistema brasileiro de classificação de solos. Brasília: Serviço de Produção de Informação, 1999.

EMPRESA BRASILEIRA DE PESQUISA

AGROPECUÁRIA - EMBRAPA. 2011. Disponível em: <www.uep.cnps.embrapa.brlsolos> Acesso em: 01 de nov. de 2011).

FELFILI, J. M. et al. Projeto biogeografia do bioma cerrado: vegetação e solos. Cadernos de Geociências, v.12, p.75-166, 1994.

FELFILI, J. M. et al. Comparação florística e fitossociológica do cerrado nas chapadas Pratinha e dos Veadeiros. In: LEITE, L.; SAITO, C. H. (Ed.). Contribuição ao conhecimento ecológico do Cerrado. Brasília:

Universidade de Brasília, 1997. p.6-11.

FELFILI, J. M. et al. Fitossociologia da vegetação arbórea. In: FELFILI, J. M.; SILVA-JÚNIOR, M. C. (Ed.). Biogeografia do Bioma Cerrado; estudo fitofisionômico na Chapada do Espigão Mestre do São Francisco. Brasília: Universidade de Brasília, 2001. p.35-56.

FELFILI, J. M. et al. Composição florística e fitossociologia do cerrado sentido restrito no município de Água Boa - MT. Acta Botanica Brasilica, v.16, n.1, p.103-112, 2002.

FELFILI, J. M. et al. Fitossociologia da vegetação arbórea. In: FELFILI, J. M.; REZENDE, A. V.; SILVA-JÚNIOR, M. C. (Ed.). Biogeografia do bioma Cerrado: vegetação e solos da Chapada dos Veadeiros. Brasília: Universidade de Brasília/Finatec, 2007. p.45-96.

Revista Árvore, Viçosa-MG, v.36, n.4, p.673-683, 2012
FONSECA, M. S.; SILVA-JÚNIOR, M. C.

Fitossociologia e similaridade florística entre trechos de cerrado sentido restrito em interflúvio e em vale no Jardim Botânico de Brasília, DF. Acta Botanica Brasilica, v.18, n.1, p.19-29, 2004.

FORZZA, R. C. et al. Síntese da diversidade brasileira. In: FORZZA, R. C. et al. Catálogo de plantas e fungos do Brasil. Rio de Janeiro: Instituto de Pesquisas Jardim Botânico do Rio de Janeiro, 2010. p.21-42.

HELLMANN, J. J.; FOWLER, G. W. Bias, precision and accuracy of four measures of species richness. Ecological Applications, v.9, n.3, p.824-834, 1999.

HILL, M. O. TWINSPAN: a Fortran program for arranging multivariate data in a ordered two-way table by classification of individual and attributes. Ithaca: Cornell University, 1979.

IMAÑA-ENCINAS, J.; PAULA, J. E.; SUGIMOTO, N. Análise fitossociológica do cerrado da Fazenda Marflora. Pesquisa Agropecuária Brasileira, v.30, n.5, p.577-582, 1995.

KOVACH. Kovach computing services. 2007. MVSP 3.13 p-Multivariate Statistical Package, 2007.

KREBS, C. J. Ecological methodology. 2.ed. Longman: Addison Wesley, 1989.

MARIMON, B. S.; VARELLA, R. F.; MARIMONJÚNIOR, B. H. Fitossociologia de uma área de Cerrado de encosta em Nova Xavantina, Mato Grosso. Boletim do Herbário Ezechias Paulo Heringer, v.3, p.82-101, 1998.

McCUNE, B.; MEFFORD, J. Multivariate analysis of ecological data. Version 3.17 . Oregon: MjM Software, 1997.

MEDEIROS, M. B.; GUARINO, E. S. G.; SILVA, G. P. Fitossociologia de um trecho de cerrado sensu stricto na bacia do rio Corumbá - Área de influência Direta do Aproveitamento Hidrelétrico Corumbá IV (GO). Brasília: Embrapa Recursos Genéticos e Biotecnologia, 2005. 21p. (Boletim de Pesquisa e Desenvolvimento, 86). 
MEDEIROS, M. B.; WALTER, B. M. T.

Fitossociologia no cerrado stricto sensu no município de Carolina, MA, Brasil. Cerne, v.14, n.4, p.285-294, 2008.

MEDINA, E.; SILVA, J. F. Savannas of northern South América: a steady state regulated by waterfire interactions on a background of low nutrient availability. Journal of Biogeography, v. 17, p.403-413, 1990.

NOGUEIRA, P. E.et al. Composição florística e fitossociologia de um cerrado sentido restrito no município de Canarana, MT. Boletim do

Herbário Ezechias Paulo Heringer, v.8, p.28-43, 2001.

PAULA, J. E.; IMAÑA-ENCINAS, J.;

SUGIMOTO, N. Levantamento quantitativo em três hectares de vegetação de cerrado.

Pesquisa Agropecuária Brasileira. v.33, n.5, p.613-620, 1998.

RATTER, J. A.; BRIDGWATER, S.; RIBEIRO, J. F. Analysis of the floristic composition of the brazilian cerrado vegetation III: Comparison of the woody vegetation of 376 areas.

Edinburgh Journal of Botany, v.60, n.1, p.57-109, 2003.
RIBEIRO, J. F.; WALTER, B. M. T. As principais fitofisionomias do Cerrado. In: SANO, S. M.; ALMEIDA. S. P.; RIBEIRO, J. F. (Ed.) Cerrado: ecologia e flora. Planaltina: Embrapa Cerrados, 2008. p.153-212.

ROSSI, C. V.; SILVA JÚNIOR, M. C.; SANTOS, C. E. N. Fitossociologia do estrato arbóreo do cerrado (sensu stricto) no Parque Ecológico Norte, Brasília, DF. Boletim do Herbário Ezechias Paulo Heringer, v.2, p.49-56, 1998.

SAPORETTI-JUNIOR, A. W.; MEIRA-NETO, J. A. A.; ALMADO, R. P. Fitossociologia de Cerrado sensu stricto no município de Abaeté-MG.

Revista Árvore, v.27, n.3, p.413-419, 2003.

SEPLAN. Atlas do Tocantins: subsídios ao planejamento da gestão territorial. Palmas: Secretaria de Planejamento, 2008. 62p.

SILVA-JÚNIOR, M. C.; FELFILI, J. M. A vegetação da Estação Ecológica de Águas Emendadas. Brasília: SEMATEC/ IEMA-IBAMA, 1998. 43p.

WALTER, B. M. T.; AQUINO, F. G. Flórula arbórea do cerrado sentido restrito na porção ocidental do território indígena Krahô, TO. Boletim do Herbário Ezechias Paulo Heringer. v.13, p.5-19, 2004. 
